\title{
Pensando la integración Chile-Perú desde una comprensión de la escuela como espacio público transfronterizo
}

\section{Thinking of Chile-Peru integration from understanding school as a cross- border public space}

\section{Eduardo Cavieres-Fernández ${ }^{1}$}

Centro de Estudios Avanzados, Universidad de Playa Ancha. Valparaíso, Chile ecavieres-cea@upla.cl

Citar como: Cavieres-Fernández, E. (2018). Pensando la integración Chile-Perú desde una comprensión de la escuela como espacio público transfronterizo. Desde el Sur, vol. 10, nro. 2, pp. 303-315.

\section{RESUMEN}

Como parte de los estudios que se han realizado acerca de la integración Chile y Perú, el presente trabajo quiere proponer la noción de una escuela como espacio público transfronterizo para pensar en cómo la educación puede ayudar al acercamiento entre niños chilenos y peruanos. No obstante, dicha noción no debe pensarse en abstracto, sino a partir de experiencias que ya se estén realizando. Al respecto, las experiencias entre tacneños y ariqueños resultan de particular interés.

\section{PALABRAS CLAVE}

Frontera, escuela, Chile-Perú

\section{ABSTRACT}

As part of the studies on the Chilean and Peruvian integration, this paper proposes the concept of schools as a cross-border public space to think about how education can help bringing Chilean and Peruvian children closer together. However, this approach should not be thought of in the abstract, but on the basis of experiences already taking place. In this regard, the experiences between Tacneños and Ariqueños are of particular interest.

1 PhD en Educación, major en Teoría Curricular y minor en Políticas Educativas por la Universidad de Wisconsin, Estados Unidos. Magíster en Educación con mención en Orientación Educacional por la Universidad Metropolitana de Ciencias de la Educación (Santiago de Chile). 


\section{KEYWORDS}

Borders, school, Chile-Peru

\section{Introducción}

Desde hace más de una década, se han desarrollado un conjunto de estudios y esfuerzos que se sitúan dentro de una temática que genéricamente se ha venido a llamar «de integración Chile y Perú» (CavieresFigueroa y Orrego, 2013). En consonancia con ellos, en este texto discuto la noción de la escuela como un espacio público de integración transfronterizo y sus aportes al desarrollo de estos estudios sobre la integración entre Chile y el Perú. A continuación, esbozo brevemente el área cubierta por estos estudios, y en donde se ubicaría la presente propuesta.

\section{Los aportes teóricos de la escuela para una integración de Chile y el Perú}

En el presente trabajo, por estudios sobre la integración de Chile y el Perú me refiero a los trabajos de un grupo de investigadores chilenos y peruanos que, bajo el liderazgo del profesor chileno Eduardo Cavieres Figueroa, reflexionan respecto de la relación que existe entre ambos países. Utilizando a la historiografía como disciplina principal, un texto fundacional lo constituye «Perú-Chile/Chile-Perú: 1820-1920. Desarrollos políticos, económicos y culturales» que resalta los puntos de contacto entre ambos países (Cavieres Figueroa y Aljovín de Losada, 2005), mientras otros trabajos ponen atención a eventos históricos como la Guerra del Pacífico, dado su impacto negativo para construir lazos de amistad entre los países (Cavieres-Figueroa y Chaupis, 2015). El conjunto de estos esfuerzos se ha ido focalizando en la influencia que los respectivos Estados, y los grupos de poder presentes en ellos han tenido sobre las relaciones entre ambos países a nivel no solo militar, sino también cultural, político y económico, y en el contexto más amplio de las relaciones entre Estados a escala mundial (Cavieres-Figueroa, 2015).

Lo anterior, a su vez, ha ido requiriendo perspectivas más comprehensivas y disciplinariamente diversas. Entre ellas, el campo de la educación aporta al tema de la integración de Chile y el Perú, precisamente porque ofrece un locus privilegiado para entender la naturaleza del Estado. Por lo pronto, los sistemas educativos no existirían si no fuesen por los Estados, que, para preservar su centro directriz dentro de la sociedad, los subordinan a la transmisión oficial de aquellos conocimientos que les son relevantes para sus propios objetivos dentro de la sociedad (Apple, 1995). Por tanto, lo que transmiten las escuelas refleja en buena medida las ideas que son funcionales al Estado. En ese sentido, poner atención a los contenidos que se transmiten en la escuela da una noción de cuánto les 
interesa a los Estados chileno y peruano promover un conocimiento que suscite integración entre ambos países (Cavieres-Fernández, 2015).

De este modo, hoy los currículos escolares en Chile y en el Perú se orientan por nociones como la modernización y el desarrollo económico, que remiten a un régimen de medición globalizado que exige a los países estandarizar sus currículos para comparar el aprendizaje de sus estudiantes y aumentar la competitividad y productividad de sus respectivos sistemas educativos (Sahlberg, 2006). En ese contexto, es difícil pensar que los contenidos curriculares puedan promover la integración de ambos países, si sus estudiantes deben competir entre sí para mejorar su posición en rankings internacionales y así acercarse a los primeros lugares ya ocupados por países de otras latitudes. Guardando las proporciones, esto no dista mucho de la racionalidad que primó en la Guerra del Pacífico y conforme a la cual, finalmente, el conflicto se sustentó en la competencia entre Chile y el Perú por alcanzar un lugar en un mercado económico ya dominado por países económicamente más poderosos (Cavieres-Figueroa y Aljovín de Losada, 2005b).

Sin embargo, sería un reduccionismo plantear que el aporte de las escuelas a la integración es escaso debido a la influencia del Estado sobre ellas. No hay que olvidar que los Estados no son uniformes en la medida en que los grupos a su interior tampoco lo son, albergando distintos objetivos y valores. En la misma elaboración de las directrices curriculares oficiales, y de los textos escolares que se ajustan a ellas, influyen grupos con diversos posicionamientos que deben ser consensuados (Apple, 2014). Ello crea espacios para que se incorporen contenidos con distintas orientaciones. Así, a partir de nuestro estudio sobre el tratamiento de la Guerra del Pacífico en los textos escolares de Chile y el Perú de la última década, notábamos que aun cuando en ellos se exculpaba a los respectivos Estados frente al conflicto, promoviendo el antagonismo; al mismo tiempo se promovía entre los alumnos una mirada reflexiva respecto del costo de la guerra con una perspectiva hacia la paz (Cavieres-Fernández, Castro, Cavieres-Figueroa y Chaupis, 2017). Ello refleja que siempre pueden pervivir elementos curriculares que sirvan a la integración.

\section{Escuela y espacio público de integración}

A medida que se han ido profundizando las temáticas sobre integración entre Chile y el Perú, se han buscado los espacios concretos para desarrollarlas. Por lo pronto, se ha buscado traspasar el ámbito propiamente académico estableciéndose conexiones con embajadas y consulados en los respectivos países, para dar mayor divulgación al trabajo realizado e influir en políticas sobre la materia. No obstante, puesto que el foco sobre 
los Estados ha llevado a reconocer las responsabilidades que les cabe en las tensiones que perviven entre Chile y el Perú, paulatinamente se ha ido enfatizando lo ciudadano. De este modo, sin desconocer la contribución de los Estados, se ha sido resaltando la autonomía de la ciudadanía para priorizar sus propias relaciones sociales y establecer mecanismos de integración en la vida cotidiana. En ese contexto, la noción de la «escuela» resulta de gran utilidad.

Para entender la vinculación de la escuela con la temática de la integración, me detengo brevemente en la noción de espacio público en donde radica lo propiamente ciudadano (McKee, 2005). En general, este espacio hace referencia a un lugar físico o simbólico en donde los ciudadanos deliberan y toman decisiones en torno a temas comunes; y que luego el Estado debe ejecutar. Por supuesto, en la práctica este espacio ha sido problemático en la medida en que ha servido como mera antesala para que quienes tienen liderazgo en temas ciudadanos pasen luego a ocupar cargos dentro de las instituciones del Estado; o para instalar temas que son relevantes solo para los grupos más privilegiados de la ciudadanía (Fraser, 1990). No obstante, el espacio público sigue constituyendo en nuestro sentido común una referencia a un espacio en donde los ciudadanos pueden conversar autónoma y críticamente. En muchos casos, el espacio público pareciera estar formado, a su vez, por un conjunto de otros espacios públicos más pequeños en donde los distintos grupos se van integrando de acuerdo con sus afinidades e intereses.

Dicha definición aplica perfectamente a la escuela que constituye en sí misma un espacio público (Giroux, 1988). Tal como lo señalé anteriormente, si bien las escuelas forman parte de sistemas educativos más amplios y, por tanto, dependientes de los Estados, ello no quita que también constituyan espacios en donde la ciudadanía, especialmente sus generaciones más jóvenes, se reúne para retransmitir y perpetuar su propio conocimiento y cultura. En estos espacios los jóvenes pueden ejercitar y experimentar formas de comprensión que están intrínsecamente vinculadas con su convivencia diaria. Todo ello contribuye significativamente para que aprendan a resolver sus conflictos y construyan juntos proyectos de colaboración mutua a través de la discusión, el dialogo y el intercambio de experiencias. Aquello apunta a esta noción de escuela como espacio público.

Sin embargo, otra limitación de estos espacios públicos es que, dada la influencia del Estado sobre ellos, también han contribuido a la formación de una ciudadanía intrínsecamente nacional. Consecuentemente, el espacio público moderno no está dispuesto para que los ciudadanos de distintos países fortalezcan sus mutuas necesidades e intereses, tal como 
ocurre frecuentemente a nivel de los Estados (Fraser, 2009). Entre Chile y el Perú esto ha sido evidente, especialmente a raíz de la Guerra del Pacífico, en que los Estados difundieron estereotipos entre las ciudadanías para fomentar su antagonismo (Klaiber, 1978). Tras la victoria chilena, los ciudadanos chilenos han sido particularmente racistas en su trato con los peruanos, como se observa en la inmigración de peruanos hacia Chile (Aravena y Silva, 2009; Márquez y Correa, 2015). No obstante, con ello no estoy asimilando un espacio público trasnacional con un buen régimen jurídico de defensa de los inmigrantes. Se trata de pensar en la posibilidad de que las ciudadanías de dos o más países pudiesen compartir un espacio público común, independiente de que haya otros espacios en que estas ciudadanías traten sus temas más particulares.

Si bien en un contexto de globalización, un espacio público transfronterizo podría ser válido para ciudadanías que se encuentran lejanas geográficamente dado el poder del Internet (Appadurai, 1996), con mayor razón debiera serlo para aquellas que se encuentran dentro de una misma región o que comparten fronteras. De ahí que la relación que existe entre las ciudadanías de Arica y Tacna nos resulta de interés al apuntar hacia un espacio público compartido que, aunque difuso, se desarrolla en la cotidianidad en la cual conviven los ariqueños y tacneños (Podestá, 2011). Más aún, dicha cercanía se ha construido históricamente a pesar de, y de resistencia a, una serie de acciones de los Estados que, particularmente con ocasión de los efectos que dejó la Guerra del Pacífico, buscaron instalar identidades políticas y culturales en la región que aseguraran la posesión/recuperación de territorio para las respectivas naciones, perpetuando, a su vez, el antagonismo entre las ciudadanías (Díaz, 2003). En este sentido, el espacio público que pervive entre Arica y Tacna constituye un referente para pensar de manera más radical la integración entre Chile y el Perú.

Desde esa perspectiva, cuando propongo que la escuela también sea un espacio público de integración transfronterizo estoy pensando en escuelas en donde los estudiantes chilenos y peruanos convivan a fin de pensar, dialogar y discutir juntos respecto de sus necesidades, historias y destinos comunes reforzando lazos de colaboración que lleven finalmente a la construcción de un espacio que sea mutuo. De allí que, si bien el aumento de la inmigración de peruanos en Chile y, por tanto, el aumento de niños peruanos en las escuelas, especialmente en el centro de Chile, ofrece una oportunidad de integración, no debería condicionar el cómo pensamos dicha integración. Eso no quita el profundo deber ético de trabajar para que en estas escuelas se superen las múltiples formas de racismo en contra de estos niños peruanos (Tijoux, 2013), pero partir 
de la constatación de que los niños peruanos son inmigrantes no ayuda a pensar en ellos como conciudadanos con los cuales los niños chilenos puedan construir en la escuela un modo de vivir ciudadano igualitario y recíproco.

Desde esa perspectiva, en los estudios sobre integración Chile-Perú resultan prometedoras las experiencias en las escuelas de Arica y Tacna al ofrecer una mejor comprensión de cómo pensar la integración. Al respecto, es importante referenciar los textos que han ido abordando este tema tales como «Chile-Perú, la historia y la escuela: conflictos nacionales, percepciones sociales» (Cavieres-Figueroa, 2006) y «La historia y la escuela: integración en la triple frontera: Bolivia, Chile y Perú» (Cavieres-Figueroa, 2016). En ellos, se recogen los testimonios de alumnos y profesores de Arica y Tacna reunidos en una serie de jornadas en donde deliberaron respecto de las dificultades que existen entre sus ciudadanías, así como reconocieron puntos de contacto sobre la base de experiencias compartidas y de tradiciones e historias familiares comunes. A partir de ellas, pensaron en actividades escolares que, obviando las tensiones entre los Estados chileno y peruano, fortalecieran las relaciones ciudadanas que se establecen a través de la frontera. Aquello apunta al ideal de espacio público transfronterizo esbozado anteriormente.

Por cierto, estas jornadas reflexivas dicen poco respecto de cuántos de estos ideales de integración han sido llevados a las escuelas mismas, más aún si se considera el rol que se les asignó en estas regiones para promover el nacionalismo de los respectivos Estados (Castro, 2004). Ello exige concentrarse más en un aspecto adicional que debe ser desarrollado en este grupo de estudio sobre integración Chile y el Perú y que se podría denominar pedagógico. Este aspecto se centra finalmente en el cómo podemos hacer integración real para que afecte al conjunto de nuestras ciudadanías de manera más sustancial. Al respecto, es fundamental considerar un último aspecto de cómo se han configurado los espacios públicos modernos, según el cual, para que se valoricen en ellos determinados temas, haciéndolos cotidianos y relevantes para nuestro sentido común, son los mismos ciudadanos los que deben comenzar a visibilizarlos (Bonham, 1996).

En ello, por cierto, las escuelas nos pueden enseñar mucho en tanto que ellas, actuando como espacios públicos, posibiliten que los jóvenes se ejerciten en estos temas hasta llegar a hacerlos cotidianos y relevantes. Al permitirlo, es evidente que desde la experiencia misma de los jóvenes haciendo integración entre ciudadanías, aprenderemos mucho de cómo se podría hacer integración en otros sectores de la sociedad. No obstante, lo más complejo es aprender cómo se podría lograr que las escuelas 
efectivamente se abran a incorporar estas temáticas. A continuación, aventuro dos perspectivas a considerar en el futuro.

\section{La escuela como espacio público de integración Chile-Perú}

La conceptualización de la escuela como espacio público de integración requiere ser desarrollado no tan solo teóricamente, sino también prácticamente. Dados los requerimientos de los Estados en materia educativa y su limitado aporte en estos temas, una propuesta como la que realizo puede quedar a un nivel meramente retórico, a no ser que considere como punto de partida algunos aspectos ya existentes en los establecimientos educativos.

En primer lugar, debemos localizar aquellos ciudadanos que en las escuelas visibilicen estos temas, como aquellos profesores ariqueños y tacneños que participaron de las jornadas reflexivas a las que hicimos referencia. No obstante, a la luz del conjunto de argumentos que hemos reseñado, esto no es tarea fácil. Así, por ejemplo, podemos citar el trabajo de Bustos y Díaz (2018), en el cual profesores del norte de Chile, si bien procuran acoger a los estudiantes inmigrantes del Perú, se aferran a concepciones que buscan asimilar a estos estudiantes a la escuela sin valorar completamente sus trasfondos y aportes culturales. También podemos referenciar el trabajo de Rottenbacher (2009) que muestra la asociación existente en profesores de Lima entre su identidad ciudadana y los acontecimientos y personajes de la vida nacional. Entre estos últimos, los que reciben mayor valoración son la devolución de Tacna al Perú y Miguel Grau. Ambos remiten a la Guerra del Pacífico, que aún genera división entre chilenos y peruanos.

Por dicha razón, y en segundo lugar, es fundamental vincularse con temáticas afines. Así, se podría enseñar críticamente la Guerra del Pacífico para superar las miradas nacionalistas del conflicto (Mondaca, Rivera y Aguirre, 2013); o enseñar las independencias nacionales resaltando la mancomunidad que en distintas épocas caracterizaron las relaciones entre chilenos y peruanos. Lo anterior remite, a su vez, a la pertinencia de implementar cambios curriculares en determinadas escuelas para introducir temáticas afines tanto para estudiantes chilenos como peruanos (Medina, 2007). Sin embargo, ello no debe desconocer que, si bien los actuales procesos de estandarización de los contenidos curriculares instauran mecanismos de competencia poco propicios para hablar de integración, probablemente es la primera vez en la historia de la educación que niños chilenos y peruanos están estudiando al mismo tiempo los mismos contenidos. Ello, por cierto, representa una oportunidad para crear vinculaciones entre estudiantes chileno y peruanos no solo en materias 
referidas a la historia sino a las matemáticas, lenguaje o ciencias naturales sin necesidad de crear currículos especiales para promover la integración.

Un área particularmente favorable para temas de integración es la Formación Ciudadana integrada al currículo de Chile y el Perú (Ministerio de Educación, 2013; Ministerio de Educación, 2017). Sus contenidos buscan fortalecer la participación de los estudiantes en la comunidad nacional en el contexto de exigencias globales que demandan un mayor respeto a los derechos humanos, a la diversidad social, y la valoración por la institucionalidad política del Estado. Todo ello tiene profundas implicancias para que estudiantes chilenos y peruanos reflexionen sobre cómo construir espacios públicos. No obstante, es necesario advertir que estas directrices, al ser oficiales, contienen elementos Estado-céntricos que dan más importancia a la participación política institucional que aquella que se desarrolla más autónomamente en la sociedad civil (Cavieres-Fernández, 2017). Igualmente, el tipo de unidad nacional que promueve, centrada en el Estado, se sustenta sobre una comprensión limitada de la diversidad que desconoce las desigualdades que en torno a ella se han creado de acuerdo con dinámicas de clase, raza y género.

Los anterior nos lleva a un aspecto más sustancial para nuestra temática, referido al hecho de que este conjunto de concepciones ciudadanas Estado-céntricas se sustentan sobre una comprensión individualista de la ciudadanía (Macpherson, 1977). Ahora bien, el individualismo tiene una larga historia en nuestras sociedades, y sería un error asimilar individualismo con egoísmo. Dentro de una comprensión individualista de la persona caben vinculaciones y compromisos profundos con la propia familia, el barrio o la patria. Lo problemático del individualismo más bien son los mecanismos que se establecen cuando surgen tensiones y conflictos entre los individuos y que se basan en la competencia, no en la colaboración (Gould, 1990). De ahí que el individualismo, contrario al estereotipo común, no está en contra de la asociación de las personas. Muy por el contrario, demanda mucho trabajo en conjunto, pero con personas que son similares o afines para diferenciarse y defenderse de aquellos con intereses diferentes. En términos ciudadanos, el mejor camino para lograr aquello es acceder al poder del Estado a través del voto político.

Sin embargo, el individualismo no tan solo promueve el antagonismo entre grupos dentro de la propia nación, sino que también lo hace, dentro de la estructura del Estado, respecto de otras naciones con las que se establecen relaciones de competencia y mutua exclusión. En ese sentido, si tenemos políticas educativas ciudadanas que promueven el individualismo desde las relaciones que establece el Estado, es fundamental reorientarlas en torno a otros modos de vivir la ciudadanía (Cavieres-Fernández, 
2014). En otras palabras, debemos comenzar a trabajar fuertemente para resistir los acentos Estado-céntricos e individualistas que subyacen en los referentes ciudadanos que se propagan tanto en Chile como en el Perú. Aquello es crucial para nuestro tema acerca de la integración en la medida en que da sentido a la hipótesis de que, si los grupos al interior de cada país fortalecen sus identidades y autonomía, resistiendo la segregación cultural y política que genera el individualismo y establecen, a su vez, relaciones de colaboración con otros grupos sociales; ello debería llevar a que los ciudadanos no tengan dificultades para establecer relaciones igualmente colaborativas con ciudadanos de otros países (Banks, 2007).

A su vez, si los énfasis sobre integración Chile-Perú se ponen en la colaboración más allá de las propias fronteras, ello desafiará la racionalidad individualista al interior de nuestros países que tanto incide en las relaciones entre los países. Por ello, es igualmente importante vincular la integración con los temas sobre la globalización que tímidamente van incorporando los currículos y que permiten miradas más regionales y latinoamericanas, poniendo un foco especial en la persistente desigualdad y exclusión de la diversidad social que resulta de la actual estructuración económica global (Juárez y Comboni, 2003). Frente a ello, los niños chilenos y peruanos podrán discutir y realizar trabajos para reflexionar en cómo construir relaciones de amistad entre las ciudadanías, a pesar de la adversidad estructural de la región. Aquello podría ser particularmente relevante para aquellos niños que, a pesar de las fronteras, comparten elementos de una identidad común y, por tanto, se inclinen más a colaborarse mutuamente (Vergara y Vergara, 2002).

\section{Comentarios finales}

En síntesis, en este texto he querido referir el tema de la integración Chile-Perú a una noción de escuela como espacio público transfronterizo planteando un horizonte en el cual ni dicha integración ni lo que la escuela debe hacer frente a ella se piense de una manera meramente instrumental, sino profundamente ciudadana. No basta con que en la escuela los niños chilenos y peruanos se conozcan más; ni siquiera que la escuela ayude a los niños chilenos y peruanos que abandonen sus prejuicios mutuos promoviendo sentimientos genéricos de paz. Desde la perspectiva que he desarrollado, si bien todo ello es importante, es insuficiente. Una escuela en cuanto espacio público transfronterizo debe aspirar a que los niños chilenos y peruanos se reconozcan como conciudadanos elaborando y construyendo juntos una mejor sociedad para ambos. Aquello permitiría superar los límites estrechos que los respectivos Estados han planteado para desarrollar la relación entre ambos países. 
A la luz de los estudios sobre integración entre Chile y el Perú, algunos elementos de espacio público transfronterizo ya están en construcción en escuelas de Arica y Tacna. Nuestra investigación a futuro deberá recoger las experiencias que allí se realizan viendo cómo pueden profundizarse y replicarse en otras zonas geográficas de nuestros países. Sin embargo, no desconocemos los recientes aportes de Mondaca, Muñoz, Gajardo y Gairín (2018), que advierten que tampoco se pueden desconocer las dificultades que aún persisten en estas escuelas, específicamente en Arica, dada la influencia del Estado y las deficiencias en sus directrices curriculares. Frente a ello, como muestran los autores, resulta de fundamental interés ampliar la mirada hacia otros sectores de la región, como los valles, en donde disminuye el peso institucional y las escuelas se transforman en espacios de convivencia pública en donde las tradiciones familiares y étnicas comunes terminan relegando a un plano secundario las respectivas identificaciones nacionales de chilenos y peruanos.

En definitiva, pensar a la escuela en estas materias como espacio público transfronterizo nos permitirá revalorizar los lazos comunes que tenemos chilenos y peruanos, y en cómo ayudarnos mutuamente a ser mejores ciudadanos. La escuela es el lugar adecuado para aprender sobre ello y para no dejar de creer en la premisa central de nuestros estudios: entre Chile y el Perú hay más cosas que nos unen que las que nos separan (González y Parodi, 2013). Este periodo de Bicentenario de nuestras independencias será propicio para seguir ahondando en el destino común que debemos seguir luchando por forjar y el rol que en él puede tener una escuela en cuanto espacio público transfronterizo.

\section{Contribución del autor}

Eduardo Cavieres-Fernández ha participado en la concepción del artículo, la recolección de datos, su redacción y aprobación de la versión final.

\section{Fuente de financiamiento}

Autofinanciado.

\section{Conflictos de interés}

El autor declara no tener conflictos de interés. 


\section{REFERENCIAS BIBLIOGRÁFICAS}

Appadurai, A. (1996). Modernity at Large: Cultural Dimensions of Globalization. Minnesota: University of Minnesota Press.

Apple, M. (1995). Education and Power. Nueva York: Routledge.

Apple, M. (2014). Official knowledge. Democratic education in a conservative age. Nueva York: Routledge.

Aravena, A. y Silva, F. (2009). Imaginarios sociales dominantes de la alteridad en la configuración de los límites etno-nacionales de la identidad chilena. Sociedad Hoy, vol. 17, pp. 39-50.

Banks, J. (2007). Educating citizens in a multicultural society. Nueva York: Teachers College Press.

Bohman, J. (1996). Public Deliberation. Pluralism, Complexity, and Democracy. Cambridge, Massachusetts: The MIT Press.

Bustos, R. y Díaz, A. (2018). Gestión de la diversidad en escuelas chilenas de frontera. Perfiles Latinoamericanos, vol. 26, nro. 51, pp. 123-148.

Castro, L. (2004). Una escuela fiscal ausente, una chilenización inexistente: la precaria escolaridad de los aymaras de Tarapacá durante el ciclo expansivo del salitre (1880-1920). Cuadernos Interculturales, vol. 2, nro. 3, pp. 57-68.

Cavieres-Fernández, E. (2014). Teachers' experiences and teaching civic engagement beyond self-regarding individualism. Teaching and Teacher Education, vol. 42, pp. 1-10.

(2015). Modernidad cultural, currículo escolar y antagonismo hacia los peruanos. Obstáculos y propuestas para avanzar hacia su superación. En E. Cavieres Figueroa y J. Chaupis (Eds.), La Guerra del Pacífico en perspectiva histórica. Reflexiones y proyecciones en pasado y presente. Arica: Universidad de Tarapacá.

(2017). Teacher counter stories to a citizenship education mega policy narrative. Preparing for citizenship in Chile. Journal of Curriculum Studies, vol. 49, nro. 4, pp. 414-436.

Cavieres-Fernández, E.; Castro, L.; Cavieres-Figueroa, E. y Chaupis, J. (2017). Textos escolares y la Guerra del Pacífico: ¿obstáculos o instrumentos para la integración de chilenos y peruanos? Revista de Pedagogía, vol. 38, nro. 102 , pp. 53-79.

Cavieres-Figueroa, E. (2015). El fallo de la Haya. Una mirada hacia el futuro. Valparaíso: Ediciones Universitarias de Valparaíso.

(2006). Chile-Perú, la historia y la escuela: conflictos nacionales, percepciones sociales. Valparaíso: Ediciones Universitarias de Valparaíso. 
(Ed.) (2016). La historia y la escuela: integración en la triple frontera: Bolivia, Chile y Perú. Arica: Universidad de Tarapacá.

Cavieres-Figueroa, E. y Aljovín de Losada, C. (Comp.) (2005). Perú-Chile/ Chile-Perú: 1820-1920. Desarrollos políticos, económicos y culturales. Valparaíso: Ediciones Universitarias de Valparaíso.

Cavieres-Figueroa, E. y Aljovín de Losada, C. (2005b). Reflexiones para un análisis histórico de Chile-Perú en el siglo XIX y la Guerra del Pacífico. En E. Cavieres-Figueroa y C. Aljovín de Losada (Comps.), Chile-Perú, Perú-Chile: 1820-1920. Desarrollos políticos, económicos y culturales. Valparaíso: Ediciones Universitarias de Valparaíso.

Cavieres-Figueroa, E. y Orrego, J. L. (Coord.) (2013). Conversaciones en Lima. La historia como instrumento de integración chileno-peruana. Valparaíso: Ediciones Universitarias de Valparaíso.

Cavieres Figueroa, E. y Chaupis, J. (2015). La Guerra del Pacífico en perspectiva histórica. Reflexiones y proyecciones en pasado y presente. Arica: Universidad de Tarapacá.

Díaz, A. (2003). Problemas y perspectivas sociohistóricas en el norte chileno: análisis sobre la «chilenización de Tacna y Arica». Sí Somos Americanos. Revista de Estudios transfronterizos, vol. 5, nro. 4, pp. 49-81.

Fraser, N. (1990). Rethinking the public sphere: a contribution to the critique of actually existing democracy. Social Text, vol. 25-26, pp. 56-80.

(2009). Scales of Justice. Reimagining Political Space in a Globalizing World. Nueva York: Columbia University Press.

Giroux, H. (1988). Schooling and the Struggle for Public Life. Critical Pedagogy in the Modern Age. Minneapolis: University of Minnesota Press.

González, S. y Parodi, D. (2013). Las historias que nos unen: episodios positivos en las relaciones peruanas-chilenas, siglos XIX y XX. Santiago de Chile: RIL.

Gould, C. C. (1990). Rethinking Democracy: Freedom and Social Cooperation in Politics, Economy and Society. Cambridge: Cambridge University Press.

Juárez, J. y Comboni, S. (2003). La educación en el proceso de integración de América Latina. Política y Cultura, vol. 20, pp. 54-77.

Klaiber, J. (1978). Los «cholos» y los «rotos»: actitudes raciales durante la Guerra del Pacífico. Histórica, vol. Il, nro. 1, pp. 27-39.

Macpherson, C. B. (1977). The Political Theory of Possessive Individualism: Hobbes to Locke. Oxford: Clarendon Press.

Márquez, F. y Correa, J. J. (2015). Identidades, arraigos y soberanías. Migración peruana en Santiago de Chile. Revista Polis, vol. 42, pp. 1-16.

McKee, A. (2005). The Public Sphere: An Introduction. Cambridge: Cambridge University Press. 
Medina, M. (2007). Historia común. Memoria fragmentada. La enseñanza de la historia en América Latina. Experiencias y reflexiones 2003-2005. Bogotá: Convenio Andrés Bello.

Ministerio de Educación (2013). Orientaciones técnicas y guiones didácticos para fortalecer la formación ciudadana. $7 .^{\circ}$ básico a $4 .^{\circ}$ medio. Santiago de Chile: Ministerio de Educación/División de Educación General.

Ministerio de Educación.

(2017). Currículo nacional de la enseñanza básica. Lima:

Mondaca, C.; Rivera, P. y Aguirre, C. (2013). La escuela y la Guerra del Pacífico: propuesta didáctica de historia para la inclusión educativa en contextos transfronterizos del norte de Chile. Sí Somos Americanos. Revista de Estudios Transfronterizos, vol. 13, nro. 1, pp. 123-148.

Mondaca, C.; Muñoz, W.; Gajardo, Y. y Gairín, J. (2018). Estrategias y prácticas de inclusión de estudiantes migrantes en las escuelas de Arica y Parinacota, frontera norte de Chile. Estudios Atacameños, nro. 57, pp. 181-201.

Podestá, J. (2011). Regiones fronterizas y flujos culturales: la peruanidad en una región chilena. Revista Universum, vol. 26, nro. 1, pp. 123-137.

Rottenbacher, J. M. (2009). Identidad nacional y la valoración de la historia en una muestra de profesores de escuelas públicas de Lima metropolitana. Liberabit, vol. 15, nro. 2, pp. 75-82.

Sahlberg, P. (2006). Education reform for raising economic competitiveness. Journal of Educational Change, vol. 7, pp. 259-287.

Tijoux, M. E. (2013). Las escuelas de la inmigración en la ciudad de Santiago: Elementos para una educación contra el racismo. Polis, Revista Latinoamericana, vol. 12, nro. 35, pp. 287-307.

Vergara, J. y Vergara, J. (2002) Cuatro tesis sobre la identidad cultural latinoamericana. Una reflexión sociológica. Revista Ciencias Sociales, vol. 12, pp. 77-92. 